\title{
BMJ Open Quality Improving the workflow of nursing assistants at a general hospital in Japan
}

\author{
Hiroki Isono, ${ }^{1,2}$ Sayuri Suzuki, ${ }^{3}$ Jun Ogura, ${ }^{4}$ Junji Haruta, ${ }^{5}$ Tetsuhiro Maeno ${ }^{6}$
}

To cite: Isono H, Suzuki S, Ogura J, et al. Improving the workflow of nursing assistants at a general hospital in Japan.BMJ Open Quality 2017;6:e000106. doi:10.1136/ bmjoq-2017-000106

- Additional material is published online only. To view please visit the journal online (http://dx.doi.org/10.1136/ bmjoq-2017-000106).

Received 5 May 2017 Revised 4 November 2017 Accepted 7 November 2017

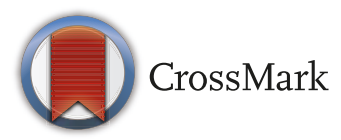

${ }^{1}$ Department of Primary Care and Medical Education, Graduate School of Comprehensive Human Sciences, University of Tsukuba, Tsukuba, Ibaraki, Japan ${ }^{2}$ Department of Medicine, Mito Kyodo General Hospital, University of Tsukuba, Mito, Ibaraki, Japan

${ }^{3}$ Department of Nursing, Mito Kyodo General Hospital, University of Tsukuba, Mito,

Ibaraki, Japan

${ }^{4}$ Department of Medical Administration, Mito Kyodo General Hospital, University of Tsukuba, Mito, Ibaraki, Japan ${ }^{5}$ Department of General Medicine and Primary Care, University of Tsukuba Hospital, Tsukuba, Ibaraki, Japan

${ }^{6}$ Department of General Medicine and Primary Care, University of Tsukuba, Tsukuba, Ibaraki, Japan

Correspondence to

Dr Hiroki Isono;

hirokisono@gmail.com

\section{ABSTRACT}

Transferring non-specialised tasks from registered nurses to nursing assistants may help registered nurses focus on specialised tasks. Optimising the workflow of nursing assistants by making their tasks more efficient may improve problems associated with the shortage of registered nurses. The nursing assistants at our hospital were stressed about referring inpatients to outpatient specialty clinics. Therefore, we initiated a project to optimise the referral process and reduce the time spent by nursing assistants on this task, with the collaboration of physicians, registered nurses and administrative assistants. The Training for Effective \& Efficient Action in Medical Service-Better Process (TEAMS-BP) method, which was developed by modifying the Japanese Training Within Industry-Job Method, was used for the optimisation process. TEAMS-BP teaches users how to break each task down into its individual components, to scrutinise the details, and then to develop new processes by eliminating, combining, rearranging and simplifying tasks. At baseline, each referral took 10 min and was performed 39 times over 10 days in six wards. The first TEAMS-BP cycle did not yield satisfactory results for the nursing assistants. In the second TEAMS-BP cycle, participants included inpatient and outpatient physicians, registered nurses and administrative assistants. As a result, we changed the referral process from paper to electronic records and streamlined referrals that were ordered by inpatient physicians to outpatient physicians. The use of this method saved the equivalent of 175 hours of nursing assistants' time per year at no additional cost. If we had been able to define the referral process as an interdisciplinary task and show the merits to each department from the beginning, we may have been able to form the interprofessional team in the first TEAMS-BP. Improving the efficiency of nursing assistants can allow other professionals to focus on their specialised tasks more effectively.

\section{PROBLEM}

This project was conducted at Mito Kyodo General Hospital in Ibaraki Prefecture, Japan. The hospital has five stories, with 320 beds in six inpatient wards on floors 2 to 5 . The outpatient clinics are on the first and second floors, located $100 \mathrm{~m}$ from the inpatient wards. In 2008, the hospital employed 22 physicians and 91 registered nurses. Following the addition of new general medicine departments through an alliance with the University of Tsukuba in 2009, staff numbers increased to 116 physicians and 250 registered nurses in
2015. Electronic medical records were introduced in 2008. The annual inpatient population also increased from 41000 to 101000 during these 8 years. The increase in the number of registered nurses relative to physicians and patients was small, however, and led to an increased workload for individual registered nurses.

The number of nursing assistants, who support registered nurses by assisting with daily tasks, remained nearly the same across the 8 years, at 20 in 2008 and 22 in 2015 . We performed an audit of nursing assistants' tasks in 2013. Their main tasks included changing diapers and linen, helping to bathe patients, cleaning rooms after discharge and replenishing supplies, with each task accounting for about $5 \%-10 \%$ of a nursing assistant's total work time. Tasks related to procuring outpatient referrals for patients (henceforth referred to as 'referrals') represented $2 \%$ of their total work time.

A referral is defined as the transfer of a consultation by an inpatient physician to an outpatient physician in a specialty clinic. In the referral process, nursing assistants in the hospital ward are required to go to the outpatient clinic and register the inpatient with reception in the morning. On the day of examination, the nursing assistant transports the inpatient to and from the outpatient clinic. Outpatients at the specialty clinic typically have a wait of 1-2 hours and inpatient examinations are performed concurrently.

In 2015, the first author (HI), a hospitalist, attended the nursing assistants' regular meeting as part of a multidisciplinary team approach. Other staff members also attended this meeting for the first time. At the meeting, the nursing assistants explained the two reasons why they felt stressed by the morning referral process: (1) they felt the process was unproductive because it required a lot of legwork to share information that was of little importance; and (2) they had to register inpatients first thing in the morning, a time when their list of tasks was already difficult to manage. However, they have continued to perform these tasks since at least 2008 because 
they felt obliged to follow the rules and did not have the means to improve the task. The first author (HI) therefore voluntarily formed a team with a nurse manager (SS, second author) and an administrative manager (JO, third author) to initiate a project to change the referral system.

Our initial aim was to reduce the time that nursing assistants spent on the referral task, focusing on time efficiency. After fully understanding the details of the referral system, however, we revised the aim to completely eliminate this task from the workflow of nursing assistants within the current fiscal year, because this would also improve the referral system for physicians, registered nurses and administrative assistants. In other words, eliminating the task was more effective than reducing the time spent on it by nursing assistants.

\section{BACKGROUND}

There is a chronic shortage of registered nurses in Japan in terms of both quality and quantity. ${ }^{12}$ Despite this shortage, registered nurses spend an average of $6.0 \%$ of their time at work completing simple tasks such as laundry, cleaning and waste disposal. Improvements to work management would therefore allow registered nurses to concentrate on their professional duties. ${ }^{3}$

In Japan, nursing assistants support the work of registered nurses and do not require certification. They perform tasks associated with improving patients' living environment, such as linen management and cleaning, and quality of life, such as helping patients eat, bathe, use the washroom and move. In addition, they perform administrative tasks, such as preparing documents and restocking supplies, in conjunction with registered nurses. $^{4}$

Since part of their work is shared, it is important that registered nurses and nursing assistants co-operate effectively. ${ }^{4}$ Registered nurses can perform more specialised tasks when nursing assistants do their jobs effectively and efficiently. ${ }^{5}$ Attempts to reduce the work load of registered nurses have included transferring some of these tasks to nursing assistants. ${ }^{6}$ As part of this, the handling of referrals in our hospital was transferred to nursing assistants. The workforce limitations of nursing assistants, however, make it important to focus on the conjoined workload of registered nurses and nursing assistants. We therefore sought to improve the referral process as a team.

\section{MEASUREMENT}

The evaluation criterion was the amount of time it took for one nursing assistant to complete the referral process. The task consisted of preparing documents in the morning, walking to the outpatient ward and registering the patient, and returning to the ward where the nursing assistant was stationed. Although we wanted to take measurements continuously, we could neither afford to hire a dedicated measurer nor automate the measurement. Furthermore, due to workload constraints, the nursing assistants declined to co-operate to make continuous measurements of referral times themselves. We therefore compromised by having each nursing assistant check the time for 10 days before the first intervention and then after every cycle of the task optimisation.

At baseline, we took 39 measurements over 10 days in all six wards in April 2015 (figure 1). The task took $10 \mathrm{~min}$ to complete.

\section{DESIGN}

We used the Model For Improvement to define the problem, aim and measurements of our intervention according to the model's three questions: (1) 'what are we trying to accomplish?', (2) 'how will we know a change is an improvement?' and (3) 'what changes can we make that will result in an improvement? ? $^{17}$

For our intervention, we used the TEAMS-BP (Training for Effective \& Efficient Action in Medical Service-Better Process) work improvement method. ${ }^{8}$ TEAMS-BP was developed at the University of Tsukuba in 2012 with the co-operation of the Japan Industrial Training Association. It is based on the Japanese Training Within Industry-Job Method (TWI-JM). As shown in online supplementary appendix 1, TEAMS-BP consists of four steps. Work improvement is carried out using the task breakdown sheet (online supplementary appendix 2 and supplementary appendix 3). Although TEAMS-BP differs from TWI$\mathrm{JM}$ in presentation, programme lectures and format, the basic concept of the four steps is the same. ${ }^{9}$

\section{Step 1}

Break down the task: the nursing assistants' task was broken down in detail and recorded on the task breakdown sheet. Several nursing assistants helped our team break down the task through careful onsite observations. Differences in how the task was conducted among nursing assistants were also noted.

\section{Step 2}

Evaluate every detailed step: once the tasks were detailed, wasteful tasks were identified and eliminated. We discussed each task as a team according to six basic questions (who, what, where, why, when and how?) and recorded the answers to these questions in the ideas column of the task breakdown sheet.

\section{Step 3}

Develop a new process: our team developed a new process when we analysed the answers generated in Step 2. Task details were individually evaluated according to the following actions, in order of eliminate, combine, rearrange and simplify. If the task detail could be eliminated, this was a $100 \%$ improvement and no further investigation was required.

\section{Step 4}

Implement the new process: we shared the new process developed in Step 3, its objective and predicted effects with the manager, colleagues and subordinates in the 


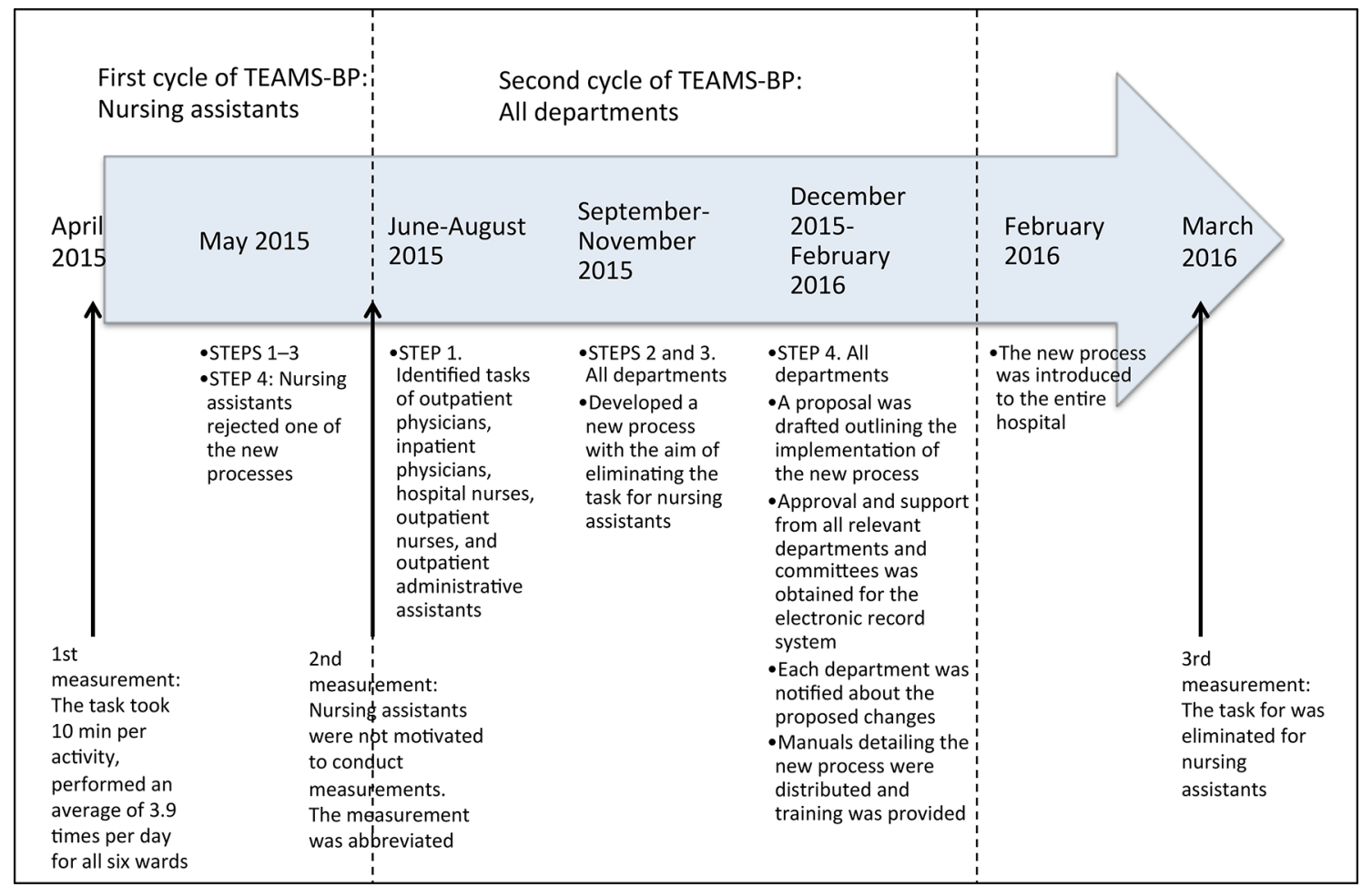

Figure 1 Summary of project team improvement efforts. TEAMS-BP, Training for Effective \& Efficient Action in Medical Service-Better Process.

relevant departments. Approvals for the safety, cost, procedure and medical aspects required for task changes were obtained from the appropriate committees before implementation. If a referral process changed, a follow-up of hearings or observations from the relevant departments was performed to examine whether or not there were problems related to the new process. In the subsequent improvement cycle, we started back at Step 1 of TEAMS$\mathrm{BP}$ to develop an even better process.

The first author (HI) first used TEAMS-BP in 2013 to improve the process of administration of tissue plasminogen activator for acute stroke, with the co-operation of physicians, registered nurses, pharmacists, technicians and administrative assistants. The first author (HI) used TEAMS-BP to clearly show the team what should be done next and to facilitate communication among the members. The project was generally considered a success and the team of professionals evaluated TEAMS-BP to be effective. A similar attempt to improve co-operation between the wards and reception within a hospital using TWI-JM was made as early as 1943 . In that case, the TWI-JM process reduced the number of movements required by administrative assistants from 29 to 10, saving 190 min of unproductive time per day. ${ }^{10}$

It was difficult to add input from nursing assistants who conducted referrals. Nursing assistants could not afford to attend the meeting because of their busy daily schedules. The nursing assistants also hesitated to change longstanding tasks, lacked motivation to make measurements associated with conducting referrals and did not participate in the investigation in 2013.

\section{STRATEGY}

TEAMS-BP was implemented as shown in figure 1.

First TEAMS-BP: we implemented the first TEAMS-BP in May 2015. We focused on the tasks of nursing assistants.

In Step 1, the team observed and recorded the nursing assistants' activities (figure 2).

In Step 2, we questioned why the identification (ID) card, which was required before the introduction of the electronic medical records system, was still being used despite the introduction of the electronic medical record to register patients. We also questioned why some nursing assistants waited in line for patient registration while others skipped the line and handed the papers to the administrative assistants; we could not identify any reason why both nursing assistants and administrative assistants were required for this task detail. We also discussed who should move between the hospital wards and outpatient clinics, and reached the consensus that one representative could collect referral papers from each nursing assistant in all six wards to reduce time. Additionally, we also sought to standardise referral processes because while some wards used a specialised referral form, others used a simple memo. Therefore, a standardised format was required across all six wards.

In Step 3, we developed four new tasks: (1) eliminate ID card use; (2) eliminate the need to wait in line at outpatient reception; (3) nominate a single representative nursing assistant to collect referral papers from all six wards and submit them to the outpatient clinic; and (4) propose the use of a standardised referral form. 


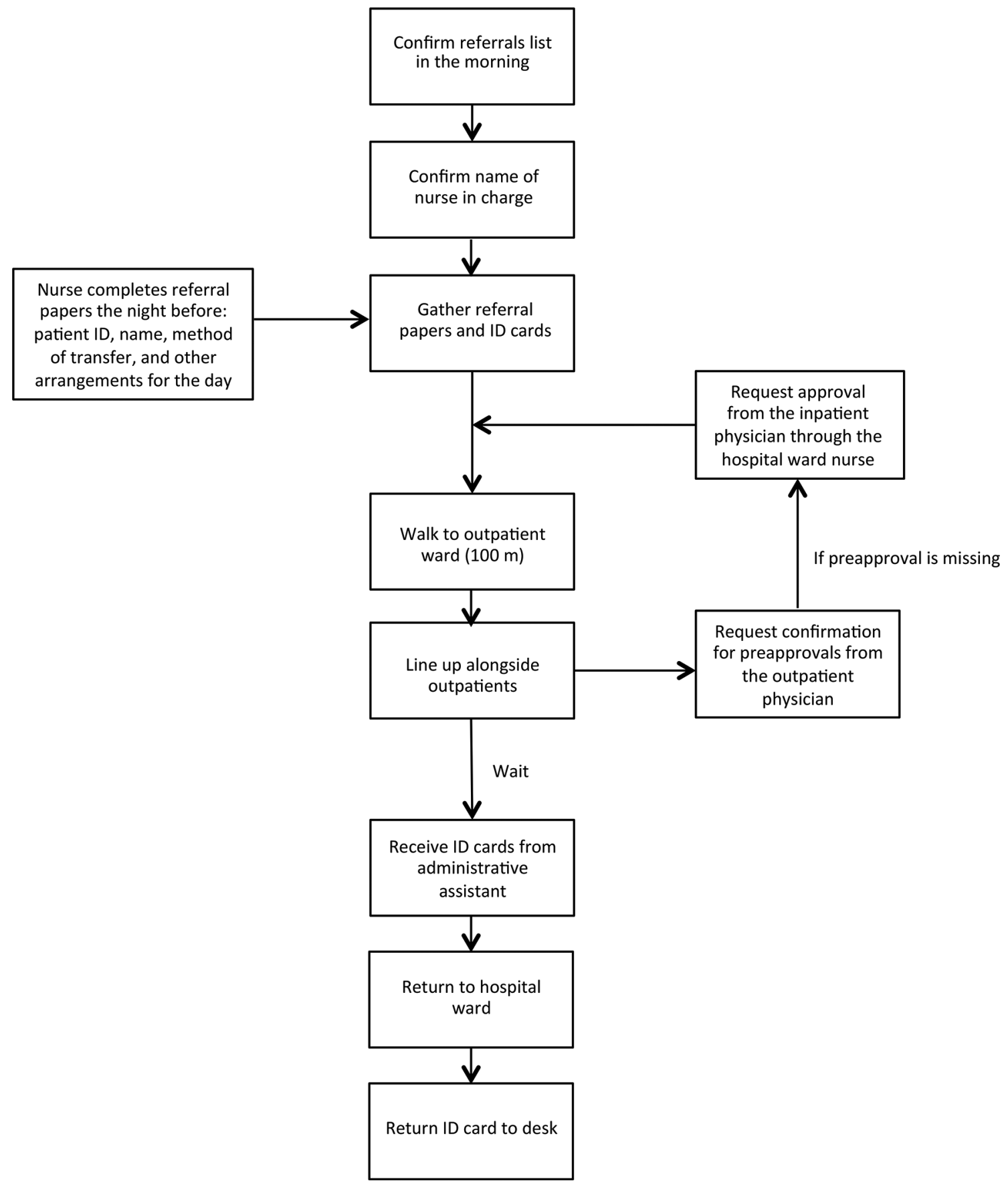

Figure 2 Activities of the nursing assistants. ID, identification.

In Step 4, we implemented items 1, 2 and 4 from Step 3 after obtaining approval from the outpatient administrative department. Item 3 was rejected by the nursing assistants because they did not want one representative to take responsibility for all six wards.

It took 1 month to reach Step 4 . We wanted to measure the time needed to complete referral registration after implementation of the first TEAMS-BP cycle. However, the changes did not result in any significant improvements for nursing assistants, and they were not motivated to conduct time measurements.

Our team made a major discovery during the first cycle. The referral process comprised three objectives: sharing information between registered nurses at the hospital and outpatient wards, ensuring that the administrative assistant registered the patient and confirming that physician approvals were obtained. We hypothesised that we could develop a better strategy to reduce nursing assistants' tasks by collaborating with other members of the hospital on the next TEAMS-BP cycle, such as physicians, registered nurses and administrative assistants. Accordingly, we abbreviated the measurement that had been planned after the first TEAMS-BP cycle because it was apparent that a second TEAMS-BP would result in a marked improvement. We subsequently performed a second TEAMS-BP. 


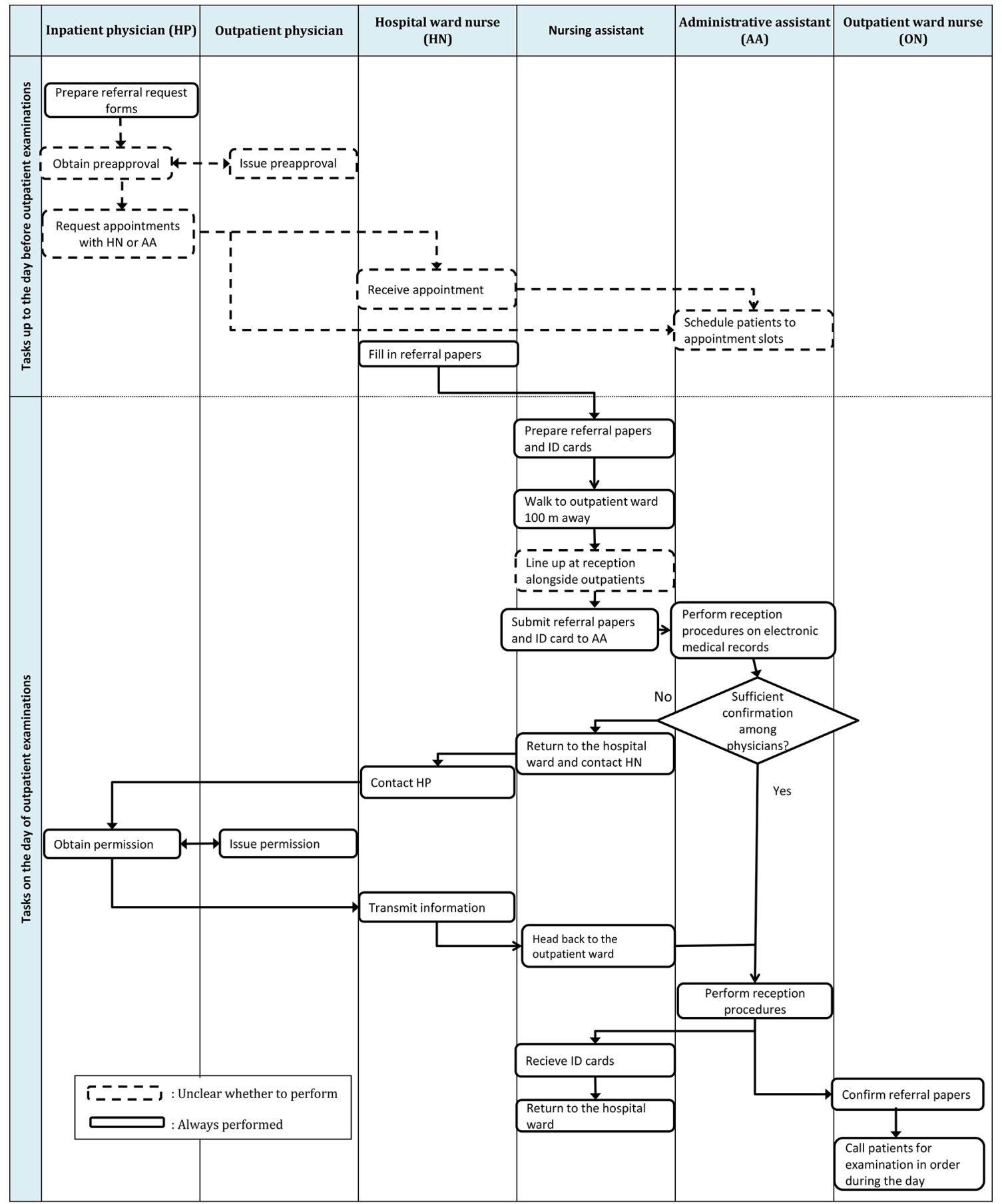

Figure 3 A baseline flowchart for the referral process. ID, identification.

\section{Second TEAMS-BP}

Our team performed Step 1 between June and August 2015. We observed the tasks of physicians, registered nurses and administrative assistants, and recorded these on the task breakdown sheet. The workflow is shown in figure 3. We found that there was no uniformity in how the task was performed. Each specialty clinic had its own rules; for example, while one clinic required the inpatient physician to personally contact the outpatient physician in advance, a second clinic did not, while a third had different rules depending on the day of the week. This was the first time that all of these rules had been systematically compared.

To develop a new process, Steps 2 and 3 were performed for each department between September and November. The project aim was changed from reducing time to eliminating the need for nursing assistants to conduct referral tasks, by collaborating between departments.

Registered nurses Steps 2 and 3: since orders to call a patient depend on his or her condition and schedule, patient information needs to be transferred from the hospital ward to the outpatient clinic. We realised that 
this information could be shared using the electronic records system. Changing the referral and registration steps to this system would eliminate the need for nursing assistants to physically transfer paper documents between wards. Patient information could be entered into the electronic records system.

Outpatient administration Steps 2 and 3: we also sought a process to improve the registration procedure. As a premise, outpatients need to make an appointment beforehand and check-in at reception on the day of examination. Since inpatients are always in the hospital, we discovered that administrative assistants could complete the reception procedures electronically without the help of nursing assistants, who have inadvertently followed a procedure that was established before the introduction of the electronic records system in 2008.

Physician Steps 2 and 3: outpatient physicians in specialty clinics had never previously discussed their referral procedures in a systematic manner. Furthermore, inpatient physicians followed vague rules to order consultations with outpatient physicians in specialty clinics. Nursing and administrative assistants had been working around this inconsistency between physicians. Both physician groups were willing to conform to a standardised process. Documentation of approval between the inpatient and outpatient physicians was required on a referral request form. Furthermore, registered nurses and administrative assistants received and processed an inpatient physician's request for consultation with the outpatient physician. Therefore, we streamlined the referral ordering process. Nevertheless, we could not eliminate all referral approval procedures between the physicians, namely in cases in which the outpatient physician wanted to make a decision at his or her own discretion depending on reimbursement, schedule and patient condition.

It took 6 months to complete Steps 2 and 3 outlined above because none of the departments were motivated to collaborate to develop on the new referral process. We explained to each department about the inefficiency of the nursing assistants' role in the referral process, and that this task could be eliminated if staff from each department made slight changes to their work. Physicians, registered nurses and administrative assistants all complied with the proposal of any new methods because they understood the current status. There had been no previous instances of interprofessional team co-operation to achieve a general improvement at our hospital. As each department was very busy with their own daily duties, the departments never gathered to discuss the development of new methods. Neither the nursing assistants nor staff in other departments wanted to have to allocate more time in their already busy schedules for making improvements.

Based on this readiness for quality improvement and the busy schedules of each of the co-operating personnel, our team visited each department separately, according to their schedules, to determine adjustments for the process. We always expressed gratitude to the staff for taking the time to meet with us, and the staff in turn welcomed us and listened to our advice. A trial to use electronic records did not increase the workload of registered nurses or outpatient administrative assistants. Therefore, we developed a uniform process that fulfilled the criteria for sharing information between inpatient and outpatient physicians, registered nurses and outpatient administrative assistants (figure 4), eliminating the task for nursing assistants.

Step 4. We created a proposal for an electronic records system that was approved by the medical, nursing, and outpatient administration departments and committees, and the new process was implemented. The proposal contained details about the new process and the expected elimination of 175 hours of work per year by nursing assistants. The 175 hours was calculated by assuming $10 \mathrm{~min}$ per activity, performed an average of 3.9 times per day for all six wards, over 270 working days per annum. After obtaining approval, we notified all relevant departments about the new process. We also created a manual detailing the process, with screenshots of the input screen for the electronic records system, to ensure correct implementation. A third cycle of TEAMS-BP was not required since the second cycle resulted in the elimination of the task for nursing assistants. We terminated the project in March 2016 (figure 1) when there were no new requests regarding nursing assistants and no issues emerged after implementation of the new process.

\section{RESULTS}

We were able to eliminate the equivalent of 175 hours from nursing assistants' work time each year by implementing two cycles of TEAMS-BP. The elimination of this task from their morning has allowed the nursing assistants to better perform their other duties, such as cleaning and taking care of patients' living environments. Furthermore, they can safely accompany patients to rehabilitation sessions every morning.

Originally, we planned to repeat the TEAMS-BP cycle and subsequent measurements. However, we were able to effectively solve the problem (the role of nursing assistants in patient referrals) after the second cycle of TEAMS-BP. Therefore, we terminated the project and considered it unnecessary to perform any postimprovement measurements.

Our results differed from the original plan and improved the task of other departments instead. We received feedback from both inpatient and outpatient physicians that standardisation of the process for referrals has made it easier to understand their mutual task. We did not receive any complaints from the physicians, registered nurses or administrative assistants to the nursing assistants or our team. We should have planned to measure outcomes such as the task times of other departments and proportion of errors before and after implementation of improvements; however, we could not foresee that the intervention would include physicians, registered nurses and administrative assistants. Despite involving multiple departments, the project did not incur any new costs. 


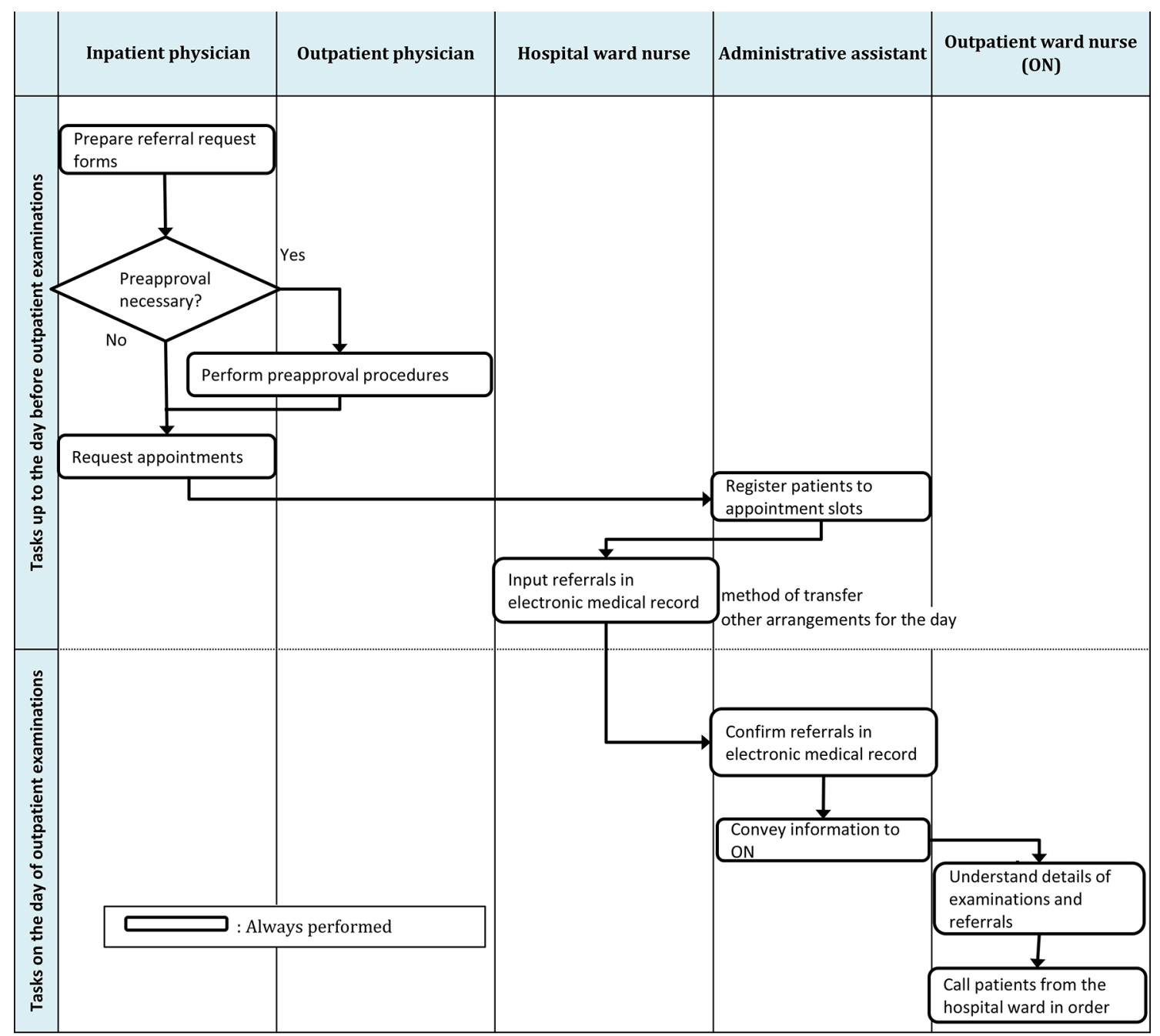

Figure 4 New flowchart for the referral process.

\section{Lessons and limitations}

This was our first project as a team and although we have improved the referral process for all current and future personnel, we cannot turn back to the clock to reclaim the hours lost unnecessarily by nursing assistants over the past 10 years. We recognise that Step 1 was a leverage point, in which we could outline the current procedure in detail and discover additional information beyond the perspective of routine work. Detailing the procedure for other departments in Step 1 of the second cycle of TEAMS-BP also led to a deeper understanding of the tasks carried out in other departments. Additionally, we learnt that the optimal route to improvement could be obtained by asking basic questions such as who, what, when, where, why and how? Developing an interprofessional team expanded our perspective on the project, which inspired ideas for improvements.

This project took 9 months to complete. The participants included inpatient and outpatient physicians, registered nurses and administrative assistants in the second cycle of TEAMS-BP. They were not aware of difficulties with the previous referral process because the nursing assistants were effective at completing the task and the details of the task were not known to others. This may explain why the participants were not motivated to advance the project. If we had been able to define the referral process as an interdisciplinary task and show the merits to each department from the beginning, we may have been able to form the interprofessional team in the first TEAMS-BP. Furthermore, it is understandable that the nursing assistants may have had difficulty committing to the project considering their position in the hierarchy of hospital personnel. They had continued to perform the referral process since at least 2008 because they felt obliged to follow the rules. Ambiguities in the approval process among physicians clearly increased the workload for nursing assistants. However, nursing assistants did not complain to the physicians nor were they recognised by the physicians for successfully conducting the approval process despite the ambiguities. Nursing assistants also accepted the fact that they had to line up for patient registration with the outpatients and could not voice their frustrations to the administrative assistants.

There are two possible reasons why physicians, registered nurses and administrative assistants were willing to alter their task procedures so readily: first, there was no 
increased workload; second, all personnel could understand the impact of an annual reduction of 175 hours of work and elimination of an entire task for nursing assistants. However, one limitation was that we could not quantitatively verify that the workload of these departments definitely did not increase. We should have collected outcomes for these departments before and after the cycle of TEAMS-BP, but we did not initially define the referral process as an interdisciplinary task when designing the study. A second limitation is that we cannot determine whether physicians, registered nurses and administrative assistants will continue to correctly implement the new process. It is possible that they may intentionally or accidentally conduct a different process, leading to the need for nursing assistants to fix the problem.

If future issues arise due to the new process, we plan to assess the task of physicians, registered nurses and administrative assistants; measure their outcomes; and conduct a new cycle of TEAMS-BP. Third, the fact that the team leader (HI) is a medical doctor may have influenced the outcome. We do not know if we would have obtained the same result if the leader of the intervention had a different occupation.

\section{CONCLUSION}

We performed two cycles of TEAMS-BP and changed the referral process among physicians, registered nurses and administrative assistants. In doing so, we eliminated 175 hours of work for nursing assistants annually without incurring additional costs. The key to the success of this project was our focus on an interprofessional team, even though the aim was limited to optimising the work of nursing assistants. Additionally, we have helped registered nurses, physicians and administrative assistants to focus on their specialised tasks, while allowing nursing assistants to more effectively take on other non-specialised tasks.

Acknowledgements The authors would like to thank Kazuto Yamaguchi of the Japan Industrial Training Association; Hideo Suzuki, Megumi Inaba, Takako
Ishitsuka, Emiko Seo, Natsuko Hayakawa of the University of Tsukuba (members of the TEAMS-BP development team); and Madoka Tsutsumi and Kazuhiro Nakazawa of the University of Tsukuba (for being our mentors).

Contributors Conception and design of study: $\mathrm{HI}, \mathrm{SS}, \mathrm{JO}$; acquisition of data: $\mathrm{HI}$, SS; analysis and/or interpretation of data: HI, TM, JH. Drafting the manuscript: HI, SS, J0; revising the manuscript critically for important intellectual content: $\mathrm{HI}, \mathrm{JH}$, TM. Approval of the version of the manuscript to be published; HI, SS, JO, JH, TM.

Competing interests None declared.

Ethics approval This study was reviewed and approved by the research ethics committee of the authors' institution.

Provenance and peer review Not commissioned; internally peer reviewed.

Open Access This is an Open Access article distributed in accordance with the Creative Commons Attribution Non Commercial (CC BY-NC 4.0) license, which permits others to distribute, remix, adapt, build upon this work non-commercially, and license their derivative works on different terms, provided the original work is properly cited and the use is non-commercial. See: http://creativecommons.org/ licenses/by-nc/4.0/

(c) Published by the BMJ Publishing Group Limited. For permission to use (where not already granted under a licence) please go to http://www.bmj.com/company/ products-services/rights-and-licensing/

\section{REFERENCES}

1. Sawada A. The nurse shortage problem in Japan. Nurs Ethics 1997;4:245-52.

2. Turale $S$, Ito $M$, Nakao F. Issues and challenges in nursing and nursing education in Japan. Nurse Educ Pract 2008;8:1-4.

3. Kudo Y, Yoshimura E, Shahzad MT, et al. Japanese professional nurses spend unnecessarily long time doing nursing assistants' tasks. Tohoku J Exp Med 2012;228:59-67.

4. Japan Industrial and Vocational Training Association. Nursing manager training text for promoting the use of nursing assistants. 2013. (published in Japanese) https://www.nurse.or.jp/home/ publication/pdf/fukyukeihatsu/kangohojyosha-text.pdf (Retrieved on 4 Apr 2017).

5. Kudo Y, Kido S, Shahzad MT, et al. Work motivation for Japanese nursing assistants in small- to medium-sized hospitals. Tohoku J Exp Med 2011;225:293-300.

6. Yatabe M, Kuroshima S, Muramori T, et al. Attempt to reduce the workload of nurses due to work transfers of nursing asssitants in hospital wards. Med J Keiju Medical Center 2012;1:8-11.

7. The Institute for Healthcare Improvement. How to improve. http://www.ihi.org/resources/Pages/Howtolmprove/Scienceoflmprov ementHowtolmprove.aspx (accessed 9 Oct 2017).

8. Maeno T. Cannot learn from clinical training! Non-technical skills for doctors. Tokyo: Yodosha, 2016:64-79.

9. Graupp P, Wrona RJ. The TWI Workbook: essential skills for supervisors: Productivity Press, 2006.

10. Adele SM. Wasted hours: job methods training pays dividends by exposing lost motion in daily routine. Hospitals 1943:47-8. 